\title{
Qui repousse un axone repousse un neurone...
}

Au cours du développement du système nerveux central, les neurones, qui naissent dans les zones ventriculaires, doivent se déplacer pour atteindre leur position définitive. La majorité des neurones migrent le long de la glie radiaire; c'est la migration dite gliophilique ou radiaire. Les autres suivent des prolongements émis par des neurones: on qualifie cette migration de neuronophilique, ou tangentielle. On connaît relativement peu de choses des mécanismes moléculaires qui permettent aux neurones en migration de s'orienter dans le tissu nerveux. Des résultats récents indiquent que des molécules diffusibles, les protéines Nétrine et Slit, impliquées dans le guidage axonal, peuvent également agir à distance sur la migration tangentielle des neurones.

\section{Slit oriente la migration tangentielle dans le télencéphale}

Chez les vertébrés, y compris chez l'homme, certains interneurones du bulbe olfactif, les cellules des grains et les cellules périglomérulaires, sont produits à distance du bulbe, dans la zone sous-ventriculaire (zsv) qui borde les ventricules télencéphaliques [1] (figure 1A). La production des neurones par la zsv débute très tardivement (vers la fin de la première semaine post-natale chez le rat) et, cas presque unique, se prolonge tout au long de la vie adulte. Ces neurones vont migrer de la zsv jusqu'au bulbe olfactif en suivant la voie de migration rostrale. Les neurones de la zsv migrent les uns sur les autres, formant ainsi des «chaînettes». La forme polysialylée de la protéine d'adhérence neuronale (PSANCAM), semble indispensable à ce processus [2]. L'identité des molécules qui orientent la migration des neurones de la zsv, du septum vers le bulbe, était jusqu'à maintenant inconnue. Cependant, on savait que le septum caudal sécrétait des facteurs diffusibles, répulsifs pour les neurones de la zsv [3]. Les travaux qui viennent d'être publiés $[4,5]$ suggèrent que les molécules impliquées appartiennent à la famille des protéines Slit $\left(\mathrm{m} / \mathrm{s}\right.$ 1999, $\left.n^{\circ} 6-7, p .882\right)$ (figure $1 \mathrm{~B}$ ). Ces dernières sont également répulsives pour des axones du bulbe olfactif, de l'hippocampe et de la moelle épinière, et elles ont pour récepteur les protéines Robo.

Par ailleurs, il vient d'être montré in vitro que les molécules Slit jouent également un rôle dans la migration tangentielle des interneurones néocorticaux GABAergiques. Ces derniers ont une origine extracorticale [6], et sont probablement issus de l'éminence ganglionnaire (EG), laquelle donne aussi naissance aux neurones du striatum (figure 1C). Des tests de confrontation en gel de collagène ont permis de montrer que la partie latérale de la zone ventriculaire de l'EG exerce une action chimio-répulsive sur les interneurones GABAergiques en migration. Cet effet répulsif peut être mimé par Slit1 et Slit2 [7]. Il reste toutefois à démontrer directement l'implication des récepteurs Robo dans le processus de migration tangentielle.

\section{La nétrine oriente la migration tangentielle dans le tronc cérébral}

Dans le bulbe rachidien ou medulla oblongata, les neurones de l'olive inférieure, ainsi que ceux à l'origine des noyaux du pont, sont produits dorsalement dans les lèvres rhombiques. Ces neurones migrent tangentiellemement à la surface du bulbe, pour atteindre leur position finale, ventrale, de part et d'autre de la plaque du plancher. Leur corps cellulaire ne traverse pas la ligne médiane ventrale, alors que leur axone la croise. Une molécule sécrétée par les cellules de la plaque du plancher, la nétrine-1, est capable d'attirer les axones commissuraux $(\mathrm{m} / \mathrm{s} 1997$, $n^{\circ} 2, p .245$ ). Il vient d'être montré qu'elle peut aussi contrôler la migration des neurones olivaires [8] et pontiques [9]. Il existe un mutant murin de la nétrine-1, obtenu par recombinaison homologue. Chez ce mutant, l'olive inférieure et les noyaux du pont sont très atrophiés et on observe de nombreux neurones en position ectopique dans le tronc cérébral. Par ailleurs, les quelques neurones olivaires qui réussissent à atteindre la plaque du plancher envoient leur axone dans la moitié ipsilatérale du cervelet, alors que normalement cette projection est controlatérale. Ces observations suggèrent que la nétrine-1 contrôle directement ou indirectement la migration, et peut-être même la survie, des neurones olivaires et pontiques. Il reste à déterminer si, dans ces systèmes, la nétrine-1 est attirante et/ou répulsive.

L'ensemble de ces données indique que les molécules diffusibles peuvent servir à orienter les cônes de croissance mais aussi à diriger la migration des neurones. Il est important de souligner que le rôle de facteurs chimiotropiques, dans le contrôle de la migration gliophilique, reste à démontrer. Toutefois, certains résultats suggèrent que, dans le néocortex des rongeurs, les cellules de Cajal-Retzius et les cellules des plexus choroïdes libèrent des protéines diffusibles susceptibles respectivement d'attirer [10] ou de repousser [4] les neurones qui migrent le long de la glie radiaire. 


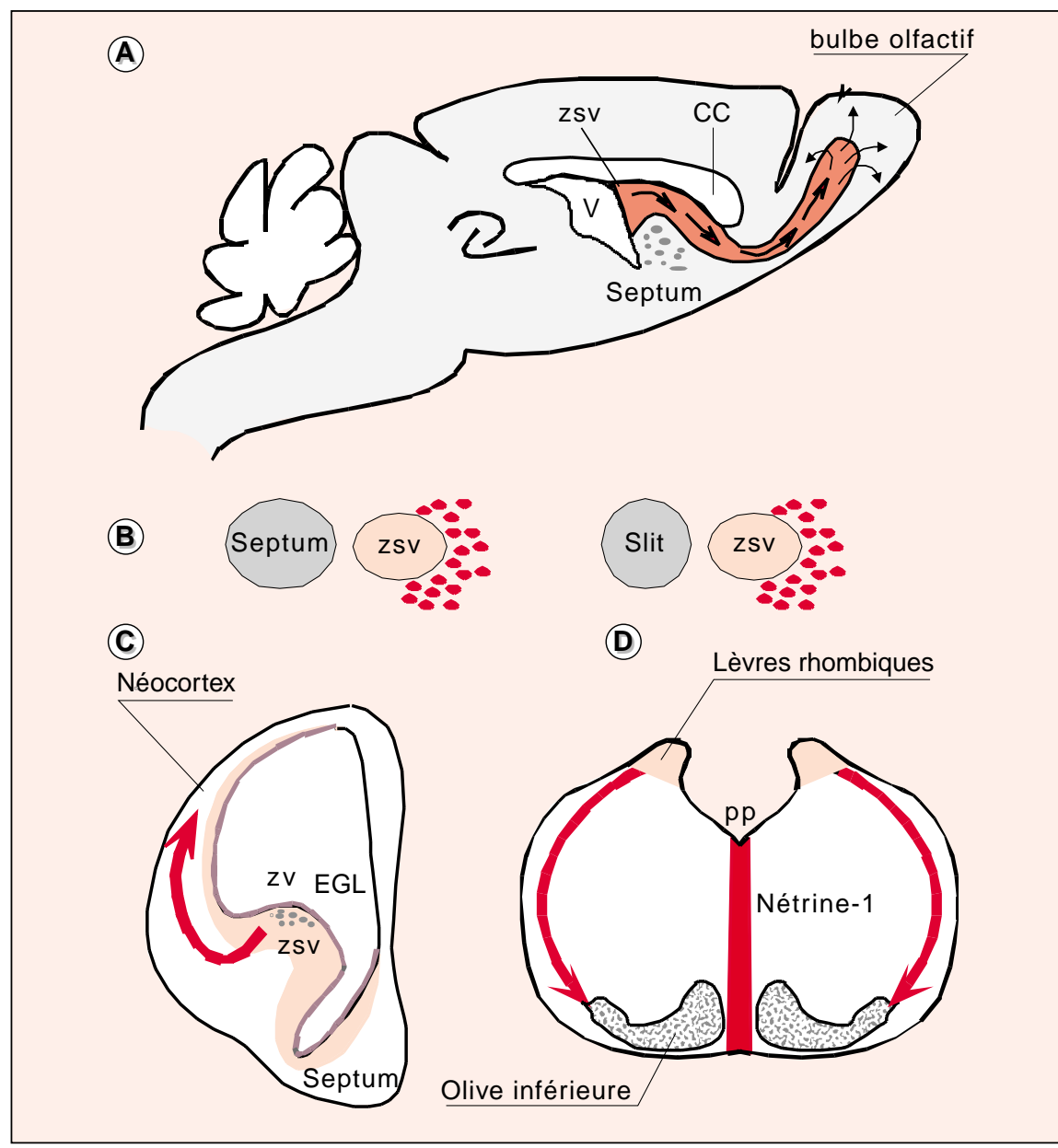

Figure 1. A. Rôle possible des protéines Slit dans l'orientation de la migration neuronale dans le cerveau de souris après la naissance. Les cellules des grains et les cellules périglomérulaires du bulbe olfactif sont produites caudalement dans la zone sous-ventriculaire (zsv) qui borde les ventricules télencéphaliques, sous le corps calleux (cc). Ces neurones migrent dans la voie de migration rostrale (en rouge) jusqu'au bulbe olfactif. Les protéines Slit1 et Slit2 (points gris foncé) sont produites par le septum et sont répulsives pour les neurones de la $z s v$. B. Schématisation des tests de confrontation en gel de collagène. Des explants de zone sous-ventriculaire sont cultivés en collagène à proximité d'explants de septum caudal (dessin de gauche) ou d'agrégats de cellules produisant Slit1 ou Slit2 (dessin de droite). Les neurones migrent préférentiellement du côté opposé au septum ou aux agrégats. $C$. Origine des interneurones GABAergiques du néocortex. Les interneurones GABAergiques du néocortex naissent dans l'éminence ganglionnaire et sont repoussés vers le cortex par les molécules Slit1 et Slit2, libérées par la région latérale de la zone ventriculaire de l'éminence ganglionnaire (EGL). D. Dans le tronc cérébral, les neurones de l'olive inférieure (en gris) et du pont sont produits dorsalement dans les lèvres rhombiques (en rose) puis migrent ventralement, tangentiellement à la surface du cerveau. Leur migration s'arrête quand ils atteignent la plaque du plancher (en rouge). La nétrine-1, produite par la plaque du plancher, contrôlerait la migration des neurones olivaires et pontiques.

1. Luskin M. Restricted proliferation and migration of postnatally generated neurons derived from the forebrain subventricular zone. Neuron $1993 ; 11: 173-89$.

2. Hu H, Tomasiewicz H, Magnuson T, Rutishauser $\mathrm{U}$. The role of polysialic acid in migration of olfactory bulb interneuron precursors in the subventricular zone. Neuron 1996; 6: 735-43.

3. Hu H, Rutishauser U. A septum-derived chemorepulsive factor for migrating olfactory interneuron precursors. Neuron 1996; 16: 933-40.

4. $\mathrm{Hu} \mathrm{H}$. Chemorepulsion of neuronal migration by slit2 in the developing mammalian forebrain. Neuron 1999; 23: 703-11

5. Wu W, Wong K, Chen JH, et al. Directional gui- dance of neuronal migration in the olfactory svstem by the protein slit. Nature $1999 ; 400$ : 331-6. 6. Anderson SA, Eisenstat DD, Shi L, Rubenstein JLR. Interneuron migration from the basal forebrain to neocortex: dependence on $D l x$ genes. Science 1997 ; 278: 474-6.

7. Zhu Y, Li HS, Zhou L, Wu JW, Rao Y. Cellular and molecular guidance of GABAergic neuronal migration from an extracortical orgin to the neocortex. Neuron 1999; 23: 473-85.

8. Bloch-Gallego E, Ezan F, Tessier-Lavigne M, Sotelo C. Floor plate and netrin-1 are involved in the migration and survival of inferior olivary neurons. J Neurosci 1999; 19: 4407-20

9. Yee KT, Simon HH, Tessier-Lavigne M, O'Leary DDM. Extension of long leading pro- cesses and neuronal migration in the mammalian brain directed by the chemoattractant netrin-1. Neuron 1999; 24: 607-22.

10. Soriano E, Alvarado-Mallart RM, Dumesnil N, Del Rio JA, Sotelo C. Cajal-Retzius cells regulate the radial glia phenotype in the adult and developing cerebellum and alter granule cell migration. Neuron 1997; 18 : 563-77.

\section{Alain Chédotal}

Inserm U.106, CHU Pitié-Salpêtrière, Bâtiment Pédiatrie, 47, boulevard de l'Hôpital, 75651 Paris Cedex 13, France. 